\title{
Hospital treatment and management in relapse of schizophrenia in the UK: associated costs
}

\author{
Janet Munro, ${ }^{1}$ Sarah Osborne, ${ }^{2}$ Lindsay Dearden, ${ }^{3}$ Katie Pascoe, ${ }^{3}$ Aline Gauthier, ${ }^{4}$ Martin Price ${ }^{3}$
}

The Psychiatrist (2011), 35, 95-100, doi: 10.1192/pb.bp.109.027714

${ }^{1}$ Optimal Medicine, London; ${ }^{2}$ King's College London; ${ }^{3}$ Janssen-Cilag;

${ }^{4}$ Amaris Group

Correspondence to Janet Munro (janet.munro@optimalmedicine.com)

First received 7 Aug 2009, final revision 10 May 2010, accepted 28 Sep 2010

\begin{abstract}
Aims and method Relapse in schizophrenia carries a heavy burden. This study aimed to describe the characteristics of patients admitted to hospital for relapse and to explore the treatment, length of stay and associated in-patient costs. The sample comprised individuals discharged from a London National Health Service trust following a relapse of schizophrenia. The costs of hospital treatment were obtained by applying referenced unit costs.
\end{abstract}

Results The cohort comprised 71 patients. Treatment non-adherence was implicated in $76 \%$ of relapses. Mean length of hospital stay was 138.9 days (range 1 week1.8 years). Mean admission cost was $£ 25852$ (range $\notin 1270-120000$ ). Over $97 \%$ of costs were hospital costs and less than $3 \%$ drug costs.

Clinical implications Hospital treatment for relapse of schizophrenia carries a considerable economic burden, as shown in this study. Treatment non-adherence is a significant factor contributing to relapse.

Declaration of interest J.M. and S.O. were employed by King's College London, Institute of Psychiatry and funded by Janssen-Cilag for their work in this project. L.D., K.P. and M.P. are employees of Janssen-Cilag.
Schizophrenia progresses according to different phases. ${ }^{1}$ Wiersma et $a l^{2}$ reported that the first psychotic episode lasts, on average, 20 months. Only $10-15 \%$ of individuals do not experience further psychotic episodes. ${ }^{2,3}$ After recovery from the first acute episode, most individuals have periods of partial or complete remission interspersed with relapses of varying length and severity. ${ }^{4}$

\section{Schizophrenia relapse: costs and risk}

Relapse in schizophrenia is a major clinical event and commonly affects the personal and social functioning of the person. Moreover, relapses are associated with a high economic burden. A study conducted in the UK showed that the 6-month direct cost of healthcare services for individuals who experience a relapse is four times higher than for those who do not (£8212 v. £1899), most of the difference being explained by in-patient costs. ${ }^{5}$

The risk of relapse for a person with schizophrenia has been estimated at $3.5 \%$ per month ${ }^{6}$ and approximately $40 \%$ experience a relapse within the year following hospital admission. ${ }^{7}$ Risk factors include poor adherence, severe residual psychopathology, poor insight, substance misuse and poor interpersonal relationships. ${ }^{6}$ It is estimated that $40 \%$ of relapses are caused by poor treatment adherence, ${ }^{8}$ a major problem in many service users. ${ }^{9}$ As relapse in schizophrenia is associated with a heavy human and financial burden, its prevention has become an important goal.

In 2002, the National Institute for Health and Clinical Excellence (NICE) issued guidance on the use of atypical antipsychotic drugs for the treatment of schizophrenia. ${ }^{10} \mathrm{It}$ recommended that individuals experiencing adverse effects or unsatisfactory response to typical medications be switched to atypical antipsychotics. In addition, the prescription of clozapine was advocated in those with treatment-resistant schizophrenia. Implementation of this guidance was expected to improve treatment adherence and shift individuals from in-patient care. ${ }^{10}$ In an updated guideline, ${ }^{11}$ NICE emphasises the importance of relapse prevention and treatment adherence: 'For people with established schizophrenia, the chance of relapse while receiving continuous antipsychotic medication appears to be about a third of that on placebo' (p. 98).

Although the cost of schizophrenia in the UK is relatively well documented, data related to the characteristics of individuals who experience relapse and its cost are scarce since the implementation of the 2002 NICE guidance. The only publication on this topic we identified is the study conducted by Almond et al, ${ }^{5}$ which compared patients' characteristics and 6-month treatment costs according to relapse status. However, as the relapse may last longer than 6 months, this estimate may not capture the total cost. 


\section{Study aims}

We aimed to assess the cost of a schizophrenia relapse admission in South London and Maudsley NHS Foundation Trust (SLAM trust). The first objective was to describe the characteristics of individuals admitted to hospital for relapse. Second, the study aimed to describe the pharmacological treatment of the relapse. The final objective was to determine the length and cost of the relapse admission.

\section{Method}

The study was approved by the local ethics committee and research and development unit. The study was conducted between 2004 and 2005. The SLAM trust provides mental health and substance misuse services to a catchment population of more than 1 million. ${ }^{12}$

The study team (research nurse or psychiatrist) identified in-patients aged 18 or older, with a diagnosis of schizophrenia or schizoaffective disorder. Diagnosis was determined using the ICD-10 codes entered as standard practice by the psychiatrist (responsible medical officer) on admission. A relapse admission was defined as readmission with a diagnosis of schizophrenia or schizoaffective disorder, thus excluding individuals experiencing their first in-patient episode. The circumstances precipitating hospital admission assessed in the study were reviewed and individuals admitted for reasons other than a schizophrenia relapse were excluded. Patients provided written consent to participate. Once the person was discharged from hospital, a retrospective analysis of medical notes was undertaken.

Information was obtained from the patients and the trust database. The sociodemographic characteristics documented were: age, gender, ethnic origin, marital status, employment status and accommodation. Medical history was recorded: age at diagnosis of schizophrenia, substance misuse, prior prescription of clozapine and number of in-patient stays for schizophrenia over the 5 years preceding the current admission. Last, the details of in-patient stay were recorded: diagnosis on admission (ICD-10), treatment at the time of admission and whether or not the person was detained under the Mental Health Act. Details of prescribed psychotropic medications were recorded from admission to discharge. For each treatment the drug name, dose, route, start date and stop date were recorded. The case notes were reviewed to identify whether non-adherence to treatment was documented to be a cause of the relapse and were classified as: 'Not known'; 'No'; 'Yes suspected'; 'Yes definite'; and 'Yes, as one of a number of factors'. No further information regarding other causes of relapse was documented.

Cost per in-patient day was estimated using the Personal Social Services Research Unit 2006 estimate. $^{13}$ Daily cost of National Health Service mental health hospital services is specific to the type of stay: acute stay (£201), long stay (£181) and psychiatric intensive care (£528). To avoid overestimating the cost of relapse, the daily cost of long stay was applied to the whole length of stay in hospital. This includes: capital costs (£25.30); salary, supplies and service costs (£99); agency overheads (£54); and disability living allowance, as calculated by the Department for Work and
Pensions (£2.30). The cost of prescribed medications was estimated using unit costs from the British National Formulary and treatment duration. ${ }^{14}$ Total cost of hospital treatment was the cost of in-patient stay plus the cost of prescribed in-patient medications.

Data analysis was undertaken using SAS version 9.1 for Windows. Descriptive statistics were generated on patients' characteristics, medical history, Mental Health Act status and prescribed psychotropic medications. Analyses were undertaken to evaluate the relationship between cost of relapse and patients' characteristics, medical history and Mental Health Act status on admission. As healthcare costs are generally positively skewed, this variable was logtransformed. Analysis of variance (ANOVA) was used to assess the effect of categorical variables and linear regression was used to test the influence of continuous variables on the total cost of relapse.

\section{Results}

A total of 112 in-patients met the eligibility criteria, of whom 71 consented to participate.

\section{Patient characteristics}

Two-thirds of patients were men $(n=47 ; 66 \%)$ and mean age on admission was 40.3 years (Table 1). More than half of the cohort was Black and minority ethnic $(n=37 ; 52 \%)$ and a quarter was White $(n=18 ; 25 \%)$. The majority of patients were single $(n=61 ; 86 \%)$ and only one patient was employed.

\section{Medical history}

The mean age at diagnosis of schizophrenia/schizoaffective disorder was 25.2 years (s.d. $=7.2$; range 16-50). Psychiatric

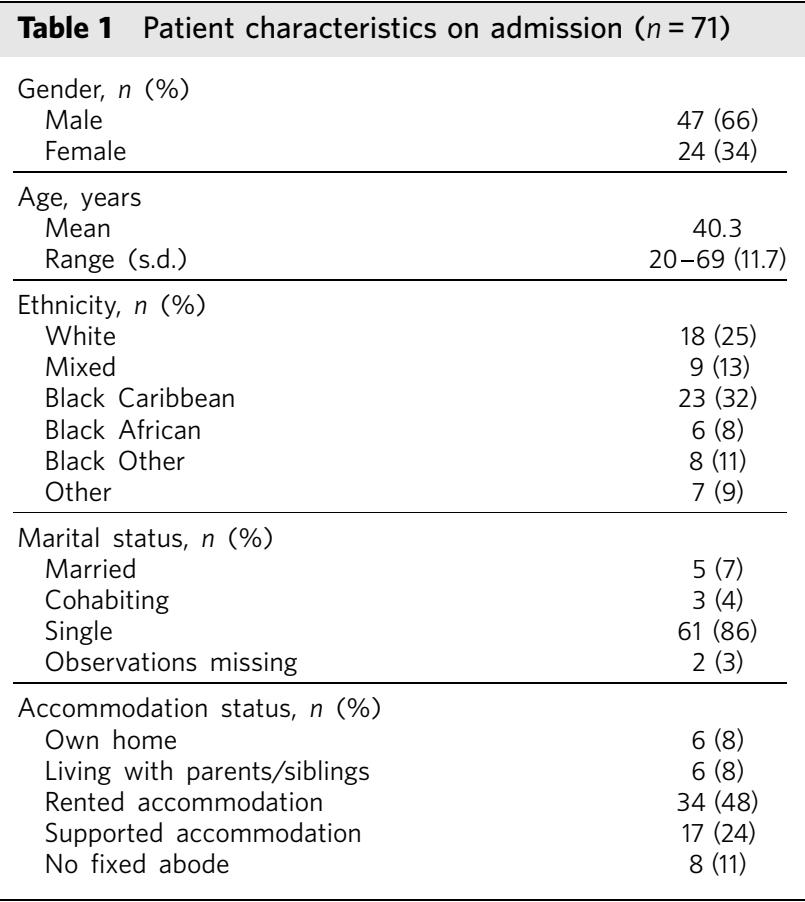


comorbidities were identified in $14 \%$ of individuals $(n=10)$ on admission. Substance misuse was reported in 27 in-patients (38\%): 21 reported using one substance only (30\%); two-thirds of these were using cannabis ( $n=14 ; 20 \%$ of the total) and fewer were using alcohol $(n=6 ; 8 \%$ of the total) or crack cocaine $(n=1 ; 1 \%$ of the total). Four patients (6\% of the total) were using multiple substances.

Almost a third of patients $(n=23 ; 32 \%)$ had received clozapine before the study admission (including those receiving clozapine on admission). All had been admitted at least once within the 5 years preceding the relapse admission; merely $11 \%(n=8)$ had been admitted only once, whereas a third had been admitted at least four times $(n=24 ; 34 \%)$.

\section{Admission for relapse}

On admission, $87 \%$ of individuals were diagnosed with schizophrenia and $13 \%$ with schizoaffective disorder. For a substantial proportion of individuals $(n=50 ; 70 \%)$ the admission was compulsory. Non-adherence to treatment was at least suspected as having precipitated admission in three-quarters of individuals $(n=54)$ : for more than a half $(n=38)$ it was recognised as a definite cause of relapse, in $14 \%$ it was a suspected factor $(n=10)$ and in $8 \%$ it was considered one of a number of factors $(n=6)$.

\section{Psychotropic medications}

\section{On admission}

At the time of admission, only a third of individuals $(n=24$; $34 \%$ ) were receiving psychotropic medications, which always included at least one antipsychotic (Fig. 1). Oral preparations were prescribed in $31 \%$ of individuals $(n=20)$ and $10 \%$ were receiving depot medications $(n=7)$, including three in-patients on both depot and oral medications.

Olanzapine was prescribed most frequently $(10 \% ; n=7)$ and sodium valproate, clozapine, quetiapine, oral risperidone and risperidone depot injection were each used in $4 \%$ $(n=3)$ of individuals on admission.

\section{On discharge}

The majority of individuals (93\%; $n=66)$ were discharged with at least one psychotropic medication, which always included at least one antipsychotic. The most common type of antipsychotic was an oral atypical used as a single agent in 21 in-patients (30\%), followed by typical depot injections as a single agent $(n=18 ; 25 \%)$. A combination of antipsychotics was prescribed in 13 in-patients (18\%) (Fig. 1).

The most often prescribed drugs on discharge were sodium valproate $(21 \%)$; clozapine $(17 \%)$; oral olanzapine (14\%); flupentixol decanoate depot (13\%); risperidone longacting injection (10\%); pipotiazine palmitate (10\%); and oral risperidone $(8 \%)$.

Among the 12 individuals who were prescribed atypical oral antipsychotics on admission, 6 were discharged on the same treatment they were admitted on (50\%), 4 switched to or added depot medication (33\%) and 2 stopped antipsychotic treatment (17\%). The two individuals on a single depot antipsychotic remained on the same treatment and the two receiving clozapine on admission were added an oral atypical antipsychotic. Other switches occurred in single cases only.

\section{In-patient length of stay and cost}

For one in-patient, information on length of stay was not available. Generally, the duration of stay varied markedly and ranged from 1 week to 1.8 years (658 days); median was 15 weeks (105 days) (Fig. 2). Mean length of stay was 138.9 days (s.d. $=133.6)$. In only $24 \%$ of in-patients $(n=17)$ length of stay was 5 weeks or less and in $24 \%$ it was longer than 6 months $(n=17)$.

The analysis of costs used log-transformed costs; the log-transformation resulted in a normal distribution of the regression residuals.

The mean in-patient cost of relapse was £25852 and varied from $£ 1270$ to almost $£ 120000$ (Table 2). More than $97 \%$ of the costs were attributable to hospital care costs and less than $3 \%$ related to pharmacological treatment. The hospital costs alone for the $24 \%$ of in-patients $(n=17)$ that were admitted for 6 months or more ranged from $£ 32942$ to $£ 119098$ and accounted for $57 \%$ of the hospital costs incurred by the whole cohort.

Factors significantly affecting the cost of relapse were analysed via univariate analyses: individuals admitted under the Mental Health Act incurred significantly higher costs compared with those admitted voluntarily, with an observed mean incremental cost of $£ 12650$ per relapse. The cost of relapse was negatively correlated with age on admission and $8 \%$ of the variation in the log-transformed costs was explained by age. Diagnosis, history of clozapine, ethnicity, gender, age at diagnosis, and 5-year history of admission and drug misuse were not significantly correlated with the cost of relapse in this cohort. As length of stay is the primary driver of cost, the relationships between in-patient characteristics and length of stay were not further explored.

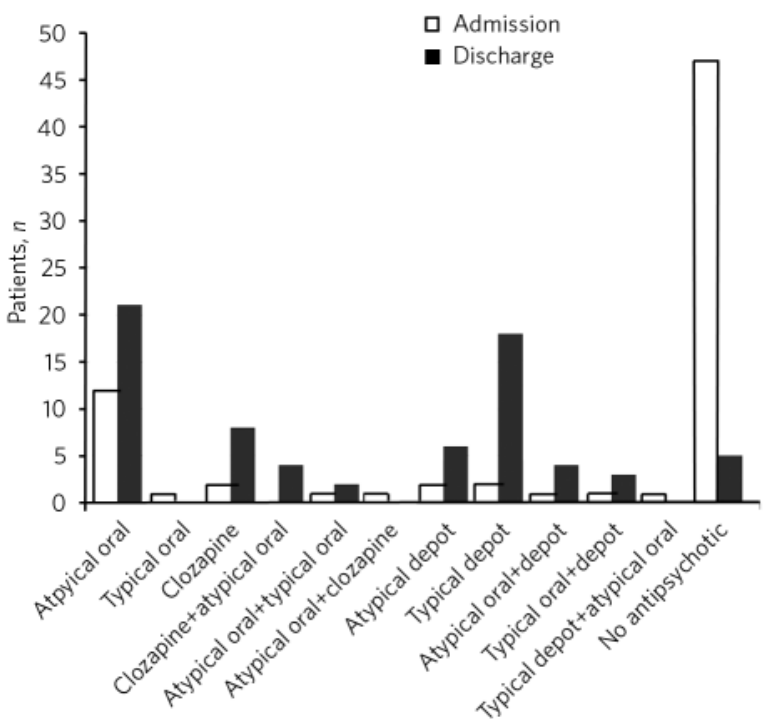

Fig 1 Antipsychotic treatments on admission and at discharge. 


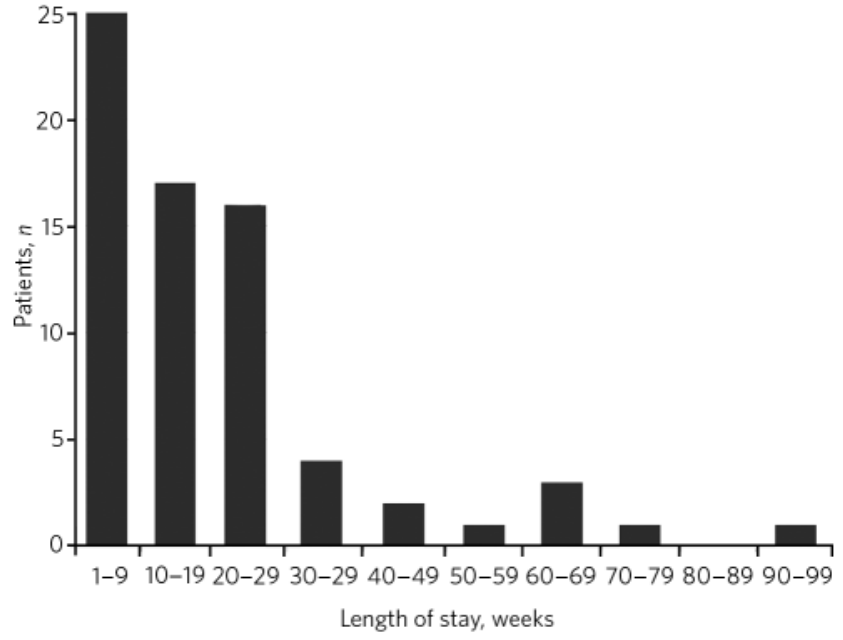

Fig 2 Hospital length of stay in weeks (mean 19.8; median 15, range 1-94)

\begin{tabular}{|c|c|c|}
\hline & Total cost & In-patient cost \\
\hline & \multicolumn{2}{|c|}{$f$} \\
\hline Mean (s.d.) & $25852(24777)$ & $25141(24186)$ \\
\hline Median & 19482 & 19005 \\
\hline Range & $1270-119933$ & $1267-119098$ \\
\hline
\end{tabular}

\section{Discussion}

\section{Main findings}

This study demonstrates the considerable economic costs of hospital admissions precipitated by a relapse in individuals with schizophrenia. It provides further insight into the extent and consequences of non-adherence to treatment, which was at least suspected to have precipitated the relapse admission in three-quarters of the cohort. Only a third of individuals were receiving antipsychotic treatment at the time of admission and of those, only a third received an antipsychotic depot preparation. Use of depot antipsychotics was markedly more frequent on discharge, when $43 \%$ of in-patients received depot. Although the duration of admission varied markedly between in-patients, the mean length of stay was 4.6 months, leading to a mean cost of hospital admission exceeding $£ 25000$ per person. The mean cost of medication was £7ll, corresponding to $3 \%$ of the hospital care budget.

\section{Study cohort}

The majority of in-patients in the study were Black and minority ethnic (52\%). The Office for National Statistics 2001 census $^{15}$ indicates that people of Black African and Black Caribbean ethnicity are in the top three ethnic groups for each borough in the SLAM trust catchment area. Moreover, the proportion of ethnic minorities in this geographical area is much higher than at the national level (34\% v. 8\%). It is worth noting that the prevalence of functional psychosis has been reported to be three times higher in Black than in White people in the UK. ${ }^{16}$

Only one individual recruited in this study had parttime employment (1.5\% of the cohort under 65 years of age). The survey conducted by Singleton et $a l^{16}$ reports that $28 \%$ of individuals with probable psychotic disorder are employed compared with $67 \%$ of individuals without such a disorder. The lower employment rate in our study may partly be explained by the local demographics and partly by the severity of illness in the enrolled population.

In the 2002 guidelines, ${ }^{10}$ NICE reported that a third of individuals with schizophrenia were unresponsive or intolerant to conventional antipsychotic drugs and should be considered for treatment with clozapine, which is consistent with the fact that $32 \%$ of the sample in our study had previously been prescribed clozapine or were on clozapine at admission. The high proportion of individuals admitted under the Mental Health Act (70\%) and the frequent in-patient episodes before the study admission suggest that individuals enrolled in this study experienced severe schizophrenia.

\section{Non-adherence to treatment}

Adherence to treatment has been identified as a main challenge in the management of schizophrenia. Weiden \& Zygmunt $^{9}$ estimated that the rate of out-patient nonadherence to maintenance antipsychotic treatment was $50 \%$ within 1 year of discharge and $75 \%$ within 2 years. Furthermore, approximately $40 \%$ of relapses are because of non-adherence. Robinson et $a l^{17}$ reported that individuals who discontinue antipsychotic therapy after a first episode of schizophrenia multiplied their risk of relapse by almost five.

Our study confirms and reinforces these findings, as non-adherence was identified as a definite cause of relapse in the majority of the sample and was at least suspected in 75\%. These proportions are consistent with the study conducted by Kamali et al, ${ }^{18}$ who reported non-adherence in two-thirds of patients re-admitted for schizophrenia or schizoaffective disorder.

Treatment adherence is influenced by multiple factors. Person-related factors are often a consequence of the illness itself, such as impaired insight into the need for medication or cognitive impairment of memory or understanding. The person's relationship with the physician and factors relating to the medication itself, such as tolerability, efficacy and dosing schedules, also influence adherence. Although only the overall contribution of non-adherence to the relapse admission could be determined from the case notes for this study, further research assessing the importance of the various risk factors may guide targeted interventions to minimise non-adherence.

Strategies for improving treatment adherence may reduce the rate of relapse. ${ }^{18,19}$ As reported in the NICE guidelines: ${ }^{11}$ 'There is also some evidence to suggest a better global outcome with depot as compared with oral antipsychotics $^{20}$ with a reduced risk of rehospitalisation. ${ }^{21}$ 


\section{Treatment pathway}

On admission, only a third of individuals received any psychotropic medication. The most commonly prescribed were oral atypical antipsychotics. On discharge, almost all in-patients (94\%) received antipsychotic treatment and the number receiving atypical oral antipsychotics had more than doubled.

Sodium valproate was among the most often prescribed medications at discharge $(21 \%)$. Although this treatment is usually used as a mood stabiliser in individuals with schizoaffective disorder, more than two-thirds of the inpatients who received sodium valproate at discharge did not have a diagnosis of schizoaffective disorder at admission. This finding reflects the diagnostic continuum of schizophrenia and schizoaffective disorder and shows that a person's diagnosis, and hence treatment, may alter over time.

Last, the number of individuals receiving antipsychotic depot medication more than quadrupled between admission and discharge, from 10 to $44 \%$, which suggests that physicians recognise the potential of depot formulations to improve outcome, possibly through better adherence.

\section{Cost of relapse}

Individuals re-admitted for a relapse of schizophrenia incurred a mean admission cost exceeding £25000, of which only $3 \%$ was attributed to medications. This study did not capture any costs associated with care after discharge, such as community mental health visits or supported accommodation, and it may therefore underestimate the total cost of a relapse involving hospital admission. The high cost of in-patient care has been documented by other authors. ${ }^{5,22}$ Almond et $a l^{5}$ reported that individuals who experienced relapse incurred 6-month treatment costs more than four times higher than those who did not (£8212 v. £1899). The definition of relapse used in their study was based on re-emergence or aggravation of psychotic symptoms and included episodes that did not require hospital treatment. The Almond study population had less severe schizophrenia than our own study cohort, as confirmed by the lower rate of admission under the Mental Health Act (20\% as compared with $70 \%$ in our study). In addition, our study captures the full in-patient treatment costs, as retrospective data capture was conducted after individuals were discharged, regardless of length of stay, whereas Almond et al report cost at a 6month cut-off. Truncating the in-patient costs at 6 months in our study resulted in a cost of approximately $£ 18900$ per patient.

Based on data from a 1994 survey report on adults living in institutions that accommodate people with mental illness, Knapp et $a l^{22}$ estimated the mean in-patient cost of schizophrenia at $£ 7487$ per annum. Jones et $a l^{23}$ analysed healthcare resource use (including hospital in-patient and out-patient services, primary and community care services, and prescribed medications) and estimated the direct cost of schizophrenia at approximately $£ 20000$ per annum.

The mean length of stay observed for the study cohort (138.9 days) was similar to the national mean; hospital episodes statistics ${ }^{24}$ report a mean length of stay of 130.2 days for 2004-2005 in individuals with schizophrenia. However, the national median (41 days) is much lower than the median observed in our study (105 days). This indicates that although hospital length of stay, and hence cost, varies widely between patients from this cohort (a quarter of patients accounting for $57 \%$ of the in-patient costs), the distribution at the national level is even more scattered, with few patients concentrating a large proportion of the costs. This reflects the NICE guideline update: ${ }^{11}$

Hospitalisation costs have been shown to drive healthcare costs incurred by people with schizophrenia, both in published evidence and in the economic analysis carried out for this guideline. It might be reasonable to argue that antipsychotic drugs that reduce the rate and length of hospital admissions (for example, drugs that reduce the rate of future relapses and/ or the length of acute episodes) are cost-saving options in the long term, despite potentially high acquisition costs. This hypothesis is supported by published evidence, which shows that increased adherence to antipsychotic treatment is associated with a significant decrease in healthcare costs incurred by people with schizophrenia through a reduction in the risk of relapse and subsequent need for hospitalisation.

This study was undertaken in the period between the publication of the two NICE schizophrenia care guidelines (2002 and 2009). Subsequent research could, therefore, focus on the treatment patterns following the publication of the 2009 guideline.

\section{Study limitations}

In this study the definition of a relapse admission was re-admission of a person with a diagnosis of schizophrenia/ schizoaffective disorder. Although admissions were screened for precipitating events other than relapse (e.g. social crises), no prospective assessments of symptom severity were available to confirm a deterioration of symptoms and a relapse of schizophrenia. However, the high level of compulsory admissions and the pressure on beds within the trust suggest that these in-patients had significant symptomatology and were not admitted for 'social' reasons. The study was based on a retrospective analysis of case notes, which inherently leads to a number of limitations. For example, the duration of historical data on prior relapse may differ between individuals. Moreover, some of the information, such as medication adherence and the reasons for any non-adherence, may not be systematically recorded during medical visits. For these parameters, the level of information and definition used may also differ between physicians. This study was conducted in the SLAM trust, whose catchment area may not be representative of all areas of the UK, as indicated by the longer median length of stay in this cohort than nationally. Therefore, the results from this cohort may not be directly representative and transferrable to other trusts in the UK, but they provide an indicative cost of an in-patient relapse. Last, the results of the study are 4 years old. However, it is important to note that this type of detailed review of schizophrenia relapse admissions in the UK setting has not been published before; the timing of the study in the context of the two NICE guideline documents is also of interest. Future research should focus on a broader 
capture of costs beyond the in-patient treatment and should assess these costs in several diverse catchment areas.

\section{Funding}

The study was funded by Janssen-Cilag. A.G. was funded by Janssen-Cilag for study analyses and preparation of study data.

\section{Acknowledgements}

We wish to acknowledge the support and encouragement of the late Professor Robert Kerwin, who was the principle investigator for this study. The study data analyses and write up were undertaken following his untimely death in 2007. We thank Jayantha Ratnayake from Quanticate for undertaking the statistical analysis and the clinical directorates and patients of the SLAM trust who participated in the study.

\section{About the authors}

Janet Munro is chief medical officer, Optimal Medicine, London; Sarah Osborne is clinical researcher and honorary specialty doctor in psychiatry, Institute of Psychiatry, King's College London; Lindsay Dearden is head of outcomes research and Katie Pascoe is senior outcomes research manager, both at Janssen-Cilag, High Wycombe; Aline Gauthier is director, Health Economics and Outcomes Research, and Amaris Group, London; Martin Price is external affairs director, Janssen-Cilag, High Wycombe.

\section{References}

1 Lamberti JS. Seven keys to relapse prevention in schizophrenia. J Psychiatr Pract 2001; 7: 253-9.

2 Wiersma D, Nienhuis FJ, Slooff CJ, Giel R. Natural course of schizophrenic disorders: a 15-year follow up of a Dutch incidence cohort. Schizophr Bull 1998; 24: 75-85.

3 McGlashan TH. A selective review of recent North American long-term follow-up studies of schizophrenia. Schizophr Bull 1988; 14: 515-42.

4 American Psychiatric Association. Diagnostic and Statistical Manual of Mental Disorders (4th edn) (DSM-IV). APA, 1994.

5 Almond S, Knapp M, Francois C, Toumi M, Brugha T. Relapse in schizophrenia: costs, clinical outcomes and quality of life. Br J Psychiatry 2004; 184: 346-51.

6 Csernansky JG, Schuchart EK. Relapse and rehospitalisation rates in patients with schizophrenia: effects of second generation antipsychotics. CNS Drugs 2002; 16: 473-84

7 Hogarty GE, Ulrich RF. The limitations of antipsychotic medication on schizophrenia relapse and adjustment and the contributions of psychosocial treatment. J Psychiatr Res 1998; 32: 243-50.

8 Weiden $\mathrm{P}$, Mott $\mathrm{T}$, Curcio N. Recognition and management of neuroleptic noncompliance in schizophrenia. In Contemporary Issues in the Treatment of Schizophrenia (eds CL Shriqui, HA Nasrallah): 411-34. American Psychiatric Publishing, 1995.

9 Weiden PJ, Zygmunt A. Medication noncompliance in schizophrenia. Part I: Assessment. J Prac Psych Behav Health 1997; 3: 106-10.

10 National Institute for Clinical Excellence. Guidance on the Use of Newer (Atypical) Antipsychotic Drugs for the Treatment of Schizophrenia (Technology Appraisal No. 43). NICE, 2002.

11 National Collaborating Centre for Mental Health. Schizophrenia: The NICE Guideline on Core Interventions in the Treatment and Management of Schizophrenia in Primary and Secondary Care (Updated Edition) (National Clinical Guideline Number 82). British Psychological Society, Royal College of Psychiatrists, 2010.

12 South London and Maudsley NHS Foundation Trust. Annual report 2004/05. SLAM, 2005 (http://www.slam.nhs.uk/about-us/ publications/annual-report.aspx)

13 Curtis L, Netten A. Unit costs of Health and Social Care 2006. University of Kent, 2006.

14 British Medical Association, Royal Pharmaceutical Society of Great Britain. British National Formulary (issue 52). BMJ Group and Pharm Press, 2006.

15 Office for National Statistics. Census 2001: Lewisham. ONS, 2003 (http://www.statistics.gov.uk/census2001/profiles/00az.asp).

16 Singleton N, Bumpstead R, O'Brien M, Lee A, Meltzer H. Psychiatric Morbidity among Adults living in Private Households, 2000: Summary Report. Office for National Statistics, 2001.

17 Robinson D, Woerner MG, Alvir JM, Bilder R, Goldman R, Geisler S, et al. Predictors of relapse following response from a first episode of schizophrenia or schizoaffective disorder. Arch Gen Psychiatry 1999; 56: 241-7.

18 Kamali M, Kelly L, Gervin M, Browne S, Larkin C, O'Callaghan E. Psychopharmacology: insight and comorbid substance misuse and medication compliance among patients with schizophrenia. Psychiatr Serv 2001; 52: 161-6.

19 Fenton WS, Blyler CR, Heinssen RK. Determinants of medication compliance in schizophrenia: empirical and clinical findings. Schizophr Bull 1997: 23: 637-51.

20 Adams CE, Fenton MKP, Quraishi S, David AS. Systematic meta-review of depot antipsychotic drugs for people with schizophrenia. $\mathrm{Br} J$ Psychiatry 2001; 179: 290-9.

21 Schooler NR. Relapse and rehospitalization: comparing oral and depot antipsychotics. J Clin Psychiatry 2003; 64 (suppl 16): s14-7.

22 Knapp M, King D, Pugner K, Lapuerta P. Non-adherence to antipsychotic medication regimens: associations with resource use and costs. $\mathrm{Br} J$ Psychiatry 2004; 184: 509-16.

23 Jones PB, Barnes TR, Davies L, Dunn G, Lloyd H, Hayhurst KP, et al. Randomized controlled trial of the effect on quality of life of second- vs first-generation antipsychotic drugs in schizophrenia: Cost Utility of the Latest Antipsychotic Drugs in Schizophrenia Study (CUtLASS 1). Arch Gen Psychiatry 2006; 63: 1079-87.

24 Hospital Episode Statistics (HESonline) Primary Diagnosis: 3 Character. HES, 2010 (http://www.hesonline.nhs.uk/Ease/servlet/ContentServer? sitelD=1937\& categoryID=203) 